University of Nebraska - Lincoln

DigitalCommons@University of Nebraska - Lincoln

3-7-2001

\title{
Contributions of genetic drift and negative selection on the evolution of three strains of wheat streak mosaic tritimovirus
}

I.-R. Choi

University of Nebraska-Lincoln

J.S. Hall

University of Nebraska-Lincoln

M. Henry

Centro Internacional De Mejoramiento de Maiz y Trigo, Mexico City, Mexico

Lingyu Zhang

University of Nebraska-Lincoln

G. L. Hein

University of Nebraska Panhandle Research and Extension Center, Scottsbluff, ghein1@unl.edu

See next page for additional authors

Follow this and additional works at: https://digitalcommons.unl.edu/plantpathpapers

Part of the Plant Pathology Commons

Choi, I.-R.; Hall, J.S.; Henry, M.; Zhang, Lingyu; Hein, G. L.; French, Roy C.; and Stenger, D.C., "Contributions of genetic drift and negative selection on the evolution of three strains of wheat streak mosaic tritimovirus" (2001). Papers in Plant Pathology. 5.

https://digitalcommons.unl.edu/plantpathpapers/5

This Article is brought to you for free and open access by the Plant Pathology Department at DigitalCommons@University of Nebraska - Lincoln. It has been accepted for inclusion in Papers in Plant Pathology by an authorized administrator of DigitalCommons@University of Nebraska - Lincoln. 


\section{Authors}

I.-R. Choi, J.S. Hall, M. Henry, Lingyu Zhang, G. L. Hein, Roy C. French, and D.C. Stenger 
Arch Virol (2001) 146: 619-628

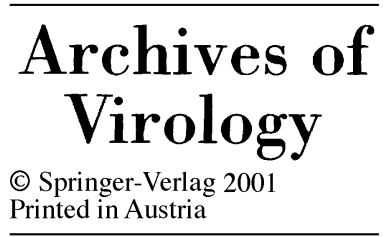

\title{
Contributions of genetic drift and negative selection on the evolution of three strains of wheat streak mosaic tritimovirus
}

\author{
Brief Report \\ I.-R. Choi ${ }^{1}$, J. S. Hall ${ }^{1}$ M. Henry ${ }^{2}$, L. Zhang ${ }^{1, *}$, G. L. Hein ${ }^{3}$, \\ R. French ${ }^{1}$, and D. C. Stenger ${ }^{1}$ \\ ${ }^{1}$ USDA-ARS and Department of Plant Pathology, University of Nebraska, \\ Lincoln, Nebraska, U.S.A. \\ ${ }^{2}$ Centro Internacional De Mejoramiento de Maiz y Trigo, \\ Mexico City, Mexico \\ ${ }^{3}$ Department of Entomology, University of Nebraska Panhandle Research \\ and Extension Center, Scottsbluff, Nebraska, U.S.A.
}

Accepted August 2, 2000

Summary. Genome sequences of three Wheat streak mosaic virus (WSMV) strains were compared. The Type and Sidney 81 strains of WSMV from the American Great Plains were closely related, with sequence identities of $97.6 \%$ (nucleotide) and $98.7 \%$ (amino acid). In contrast, the El Batán 3 strain from central Mexico was divergent, and shared only 79.2-79.3\% (nucleotide) and $90.3-90.5 \%$ (amino acid) sequence identity with Type and Sidney 81. All three WSMV strains were serologically related, however the El Batán 3 capsid protein $(\mathrm{CP})$ had 15 fewer amino acid residues. Phylogenetic analysis of the $\mathrm{CP}$ cistron indicated that Type, Sidney 81, and nine other American isolates of WSMV were closely related and distinct from the El Batán 3 sequence. Nucleotide substitutions among the WSMV strains were not randomly distributed across the genome with more variation within P1, HC-Pro, and CP, and less within P3. One 400-nucleotide region of the genome, corresponding to the $3^{\prime}$-end of $\mathrm{P} 3$, was strikingly deficient in silent substitutions. Nonetheless, the ratio of synonymous to non-synonymous substitutions throughout the genome was essentially the same for all three WSMV strains. Collectively, our data indicate that both genetic drift and negative selection have contributed to the evolution of WSMV strains.

*Pioneer Hi-Bred International, Johnston, IA 50131, U.S.A. 
Wheat streak mosaic virus (WSMV) is the type species of the recently established genus Tritimovirus of the family Potyviridae [30]. The genus Tritimovirus includes WSMV and Brome streak mosaic virus (BrSMV) $[12,13]$. Both tritimoviruses are transmitted by eriophyid mites to monocotyledonous hosts and are phylogenetically distinct from eriophyid mite-transmitted viruses in the genus Rymovirus [14, 27, 30]. WSMV has a genome organization similar to that of other monopartite members of the family Potyviridae, encoding a polyprotein that is subsequently cleaved by viral-encoded proteinases into 8-10 mature proteins capable of complex protein-protein interactions [6].

WSMV occurs throughout the major wheat growing areas of the United States and Canada, and in epidemic years may contribute to substantial losses [2]. The complete nucleotide sequence (GenBank accession AF057533) of the Sidney 81 strain of WSMV from Nebraska has been determined [30]. The Type strain of WSMV from Kansas [20] may be distinguished from Sidney 81 by the inability of Type to infect the inbred maize line SDP2 [5]. The El Batán 3 strain recently has been isolated from the Central Highlands of Mexico [28]. All three strains are efficiently transmitted from wheat to wheat by the eriophyid mite Aceria tosichella (Keifer) [2, 5, 28]. In this communication, we report the complete nucleotide sequences of the WSMV Type (GenBank accession AF285169), and El Batán 3 (GenBank accession AF285170) strains. We further examine the extent and distribution of sequence divergence among the three strains to infer major forces driving WSMV strain evolution.

Type and Sidney 81 are well characterized strains of WSMV. El Batán 3 was initially diagnosed as a WSMV strain based on symptom expression, eriophyid mite transmission, and serology [28]. Confirmation of El Batán 3 as a strain of WSMV was accomplished by reverse transcription-polymerase chain reaction (RT-PCR) of the CP cistron amplified from a total RNA sample using primers described previously $[9,21]$. The resulting PCR product was slightly smaller in size than those obtained for Sidney 81 or Type (data not shown). Western blotting of total soluble protein extracted [3] from wheat infected with each WSMV strain revealed that the $\mathrm{CP}$ of all three strains reacted to antibodies raised to Type $\mathrm{CP}$ and that the El Batán $3 \mathrm{CP}$ appeared to be of smaller size relative to the $\mathrm{CP}$ of Type and Sidney 81 (Fig. 1).

Virions of Type and El Batán 3 were purified from wheat (Triticum aestivum L.) cv. 'Centurk' as described [18], with modifications indicated [30]. Viral RNA of each strain was purified and used as templates for oligo dT primed reverse transcription [30]. Second strand synthesis was accomplished with the Klenow fragment of DNA polymerase I and Not I/EcoR I adapters were ligated to the dsDNA products.

High molecular weight ( $>9 \mathrm{kbp}$ ) dsDNA products of El Batán 3 were gel purified and ligated to Not I digested pACYC-177N1 (pACYC-177 from New England Biolabs with the unique Sma I site modified to a Not I site) and transformed into E. coli strain JM109. pWSMV-M10-11 contained a $~ 9.3 \mathrm{kbp}$ cDNA insert of the El Batán 3 genome (nts 23-9339), lacking only 22 nucleotides of the $5^{\prime}$-end. The complete nucleotide sequence of pWSMV-M10-11 was deter- 


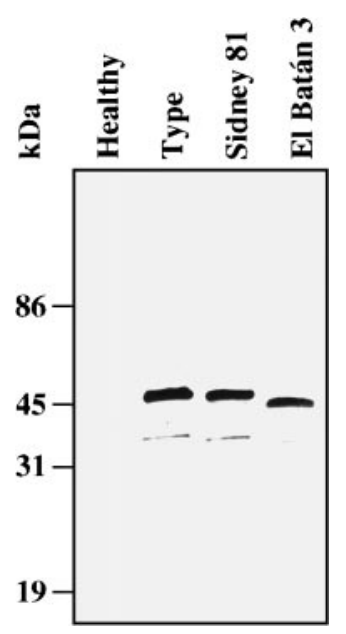

Fig. 1. Serological relatedness of the coat protein $(\mathrm{CP})$ of three Wheat streak mosaic virus (WSMV) strains. Presented is a western blot of total soluble protein samples $(20 \mu \mathrm{g})$ extracted from healthy wheat or wheat infected with WSMV strains Type, Sidney 81, or El Batán 3. The blot was probed with antibodies raised to the $\mathrm{CP}$ of WSMV-Type. The mobility and size $(\mathrm{kDa})$ of prestained protein size markers are indicated at left. Note that the CP of El Batán 3 has increased mobility relative to the other two strains. Minor bands of less than full length represent degradation products of the WSMV CP commonly observed during infection [3]

mined for both strands by a primer walking strategy using two primers annealing to regions of pACYC-177N1 flanking the insert and 40 custom oligonucleotide primers to obtain internal sequence.

Type dsDNA products contained a prominent product of $\sim 2 \mathrm{kbp}$ in size, and only traces of larger products. These were size selected $(2-4 \mathrm{kbp})$ on an agarose gel, ligated to Not I digested pGEM5zf+, and transformed into E. coli JM109. The 3'-end sequence of the Type genome was determined from four cDNA clones produced by this procedure. To obtain additional Type cDNA clones, RT-PCR using the Expand High Fidelity PCR System (Boehringer-Mannheim, Indianapolis, IN) was performed on a total RNA sample from infected wheat. First strand cDNA was prepared by reverse transcription with both oligo $\mathrm{dT}$ and random hexamer primers included in the reaction to ensure complete coverage of the Type genome. PCR was performed using primers based on the Sidney 81 sequence to yield three overlapping products (nts 1-3172, 2339-6691, and 5414-9384) that were ligated into pGEM-T (Promega) and transformed into E. coli strain JM 109. Two clones from each PCR product were sequenced completely on both strands by primer walking. Sequence discrepancies between pairs of PCR-derived clones were resolved by obtaining sequence of a third clone for each region in question.

$5^{\prime}$-end sequences of both El Batán 3 and Type were determined from three clones each produced using the 5'/3' RACE kit (Promega, Madison, WI) as described [30]. All sequencing was performed by the Iowa State University DNA Sequencing Facility, Ames, IA. Complete nucleotide sequences were compiled using the Sequencher 3.1 program (Gene Codes, Ann Arbor, MI). To eliminate potential errors resulting from mismatches between Type cDNA and Sidney 81derived primers, these regions were trimmed prior to compilation of the Type sequence. Open reading frames (ORFs) were identified using the DNA Inspector IIe program (Textco, West Lebanon, $\mathrm{NH}$ ).

The complete nucleotide sequences of Type and El Batán 3 were 9384 and $9339 \mathrm{nts}$, respectively, exclusive of the variable-length polyadenylated $3^{\prime}$-end. Conceptual translation revealed each encoded a single polyprotein ORF typical 
Table 1. Sequence identity and substitution rates among three Wheat streak mosaic virus strains

\begin{tabular}{lcclll}
\hline \multirow{2}{*}{ Strain } & \multicolumn{2}{c}{ Sidney 81} & & \multicolumn{2}{c}{ Type } \\
\cline { 2 - 3 } \cline { 5 - 6 } & \% Identity & $\mathrm{ks} / \mathrm{ka}$ & & \% Identity & $\mathrm{ks} / \mathrm{ka}$ \\
\hline Sidney 81 & $100(100)$ & - & & - \\
Type & $97.6(98.7)$ & $0.0886 / 0.0058$ & & $100(100)$ & - \\
El Batán 3 & $79.2(90.5)$ & $0.7357 / 0.0561$ & & $79.3(90.3)$ & $0.7364 / 0.0591$ \\
\hline
\end{tabular}

First number indicates percent nucleotide identity; numbers in parentheses indicate percent amino acid identity. Synonymous (ks) and nonsynonymous (ka) rates are also given for each pairwise comparison

of monopartite members of the family Potyviridae. Complete nucleotide and polyprotein sequences of Sidney 81, Type, and El Batán 3 were aligned using Clustal X [33] and compared in Table 1. The Sidney 81 and Type genomes were closely related at both the nucleotide and protein level, whereas the El Batán 3 sequence diverged from both Type and Sidney 81. The position (between nts 8272 and 8273) of a 45 nt gap in the El Batán 3 sequence, relative to Sidney 81 and Type, was determined based on the single gap in aligned polyprotein sequences and the Clustal X nucleotide sequence alignment output was manually adjusted accordingly. This $45 \mathrm{nt}$ gap encodes 15 amino acid residues within the CP cistron, and accounts for the smaller sizes of the El Batán 3 CP PCR product and CP described above. Sequencing of the El Batán 3 CP PCR product (data not shown) revealed the same $45 \mathrm{nt}$ gap as in pWSMV-M10-11. Collectively, the occurrence of identical sequence gaps in two independent clones and the apparent smaller size of the El Batán 3 CP (Fig. 1) strongly indicate that the $45 \mathrm{nt}$ gap in the El Batán 3 sequence is not an artifact of cloning. The $45 \mathrm{nt}$ gap occurs within a serine-glycine rich region, positioned 28 amino acid residues downstream of the NIb-CP junction cleaved by NIa proteinase [30] to produce mature CP. This gap could reflect a deletion unique to El Batán 3, or an insertion in the common ancestor of Type and Sidney 81 after separation from the El Batán 3 lineage.

CP nucleotide sequences of Type, Sidney 81, and El Batán 3 were aligned with CP sequences of nine additional American isolates of WSMV [4, 22] and BrSMV [13]. Because the WSMV CP sequences of Chenault et al. [4] do not include the $5^{\prime}$-terminal sequences of the $\mathrm{CP}$ cistron, sequences were trimmed to the region (corresponding to Sidney 81 nts 8369-9238) available for all taxa. A maximum likelihood distance tree was generated using the Puzzle 4.02 program [31] and the HYK model of substitution with other parameters set at default values. The unrooted tree was visualized using the TreeView program [24]. The resulting phylogenetic analysis (Fig. 2) indicated that the CP cistrons of Type and Sidney 81 were closely related to all other American isolates, and confirmed that Type and Sidney 81 are representative of genotypes common in the United States. The branch bearing El Batán 3 was longer (reflecting genetic distance) and 


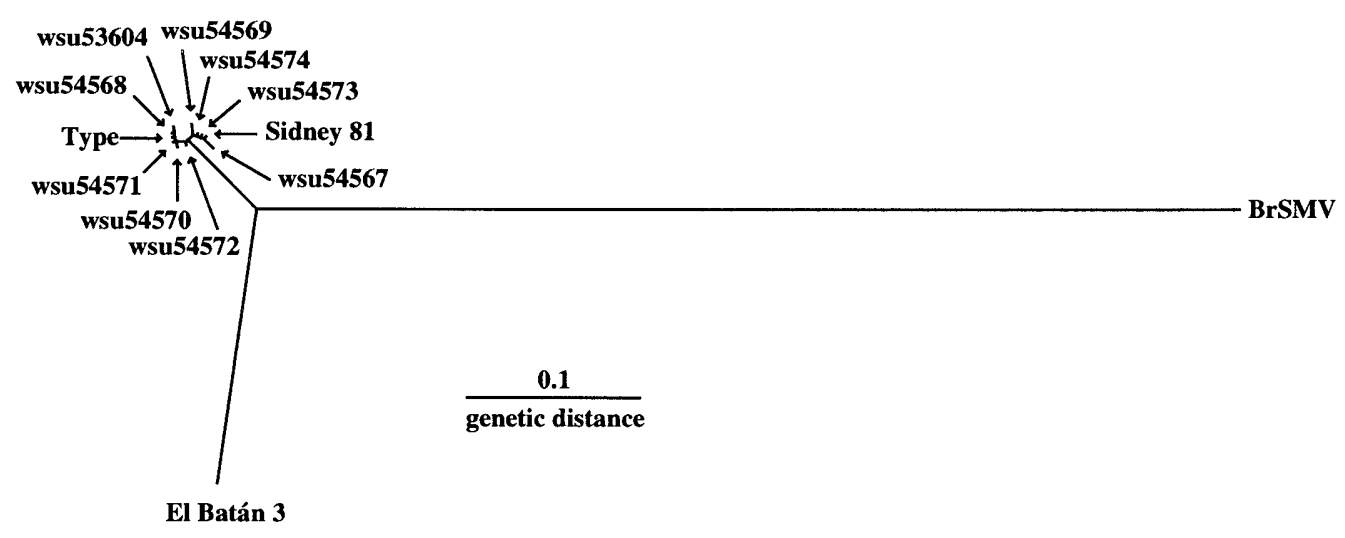

Fig. 2. Phylogenetic relationships among strains and isolates of Wheat streak mosaic virus (WSMV). Presented is a maximum likelihood distance tree, constructed using the HYK model of the Puzzle program, depicting the relationship of coat protein (CP) cistron nucleotide sequences for WSMV strains Type, Sidney 81, and El Batán 3 with CP sequences determined $[4,22]$ for nine other American isolates of WSMV. The CP cistron of the distinct tritimovirus Brome streak mosaic virus (BrSMV) was included for comparison. Branch lengths are scaled to reflect genetic distance. Support values calculated for all nodes were $\geq 67 \%$ (not shown)

separate from branches emanating from the node exclusively shared by all American WSMV taxa. Nonetheless, El Batán 3 is more closely related to American WSMV genotypes than to the European tritimovirus species BrSMV (Fig. 2).

Prior to this study, all WSMV isolates examined at the molecular level shared high sequence identity [4, 22]. Analysis of genetic variation among field isolates of WSMV from Nebraska [21] and the Texas Panhandle (R. French, unpublished) indicated that WSMV genotypes occurring in the Great Plains comprise a single population. The identification of El Batán 3 as a divergent strain of WSMV occurring in a geographically isolated area suggests a second WSMV population in North America. WSMV also is reported to occur widely in the Old World [7, $8,16,19,23,25,29,34]$. Unfortunately, there are no sequences available for any Old World isolate of WSMV, thus relationships among New and Old World WSMV populations remain undefined.

The distribution of amino acid substitutions in the polyprotein sequences of the three WSMV strains was examined using the Sequence Similarity Presenter program [10] with a window size of 4 residues and a window shift of 1 residue. The Sidney 81 polyprotein sequence served as the reference sequence for all two-way comparisons. The polyprotein sequence of BrSMV [13] was included in the analysis to contrast intra- and inter-species differences. Synonymous (ks) and non synonymous (ka) nucleotide substitution rates were calculated using the Sites computer program [15] and their distribution along the aligned WSMV genomes were analyzed by a sliding window approach implemented in the program DnaSP 3.0 [26]. The same program was used for Chi square tests of $2 \times 2$ contingency tables of $\mathrm{ks} / \mathrm{ka}$ and transitions/transversions ratios. 


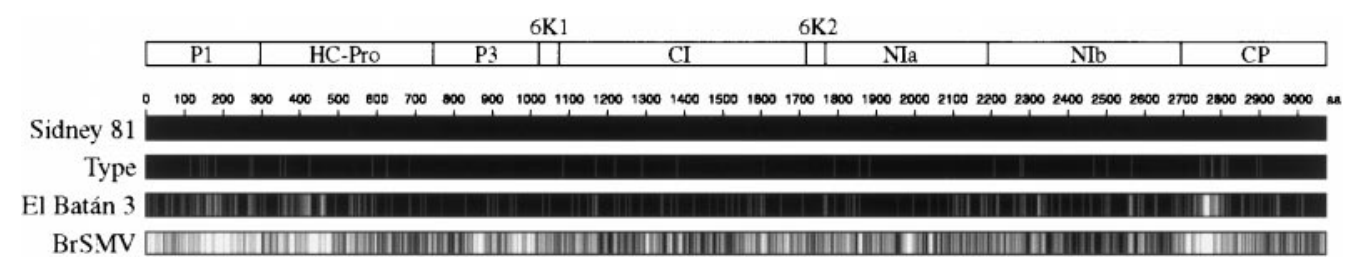

Fig. 3. Distribution of amino acid substitutions in the Wheat streak mosaic virus (WSMV) polyprotein. Presented is an alignment of polyprotein sequences of three WSMV strains (Sidney 81, Type, and El Batán 3) and the distinct tritimovirus Brome streak mosaic virus (BrSMV). The organization of the polyprotein is presented above. The analysis was conducted using the Sequence Similarity Presenter program [10] using a window size of 4 and a window shift of 1 amino acid residues. The amino-terminal portion of the BrSMV P1 protein sequence that is not present in WSMV was excluded from the analysis. Each polyprotein was compared to Sidney 81 . Shading denotes the degree of similarity within each window; ranging between black (4/4 amino acid residue matches per window) and white (0/4 amino acid residue matches per window)

Amino acid substitutions among Sidney 81, Type, and El Batán 3 were distributed along the entire polyprotein with the P1, HC-Pro, and CP cistrons being most variable (Fig. 3). Similar patterns of intraspecies variation have been observed among strains of the potyviruses Potato virus A (PVA) [17] and Yam mosaic virus [1]. There were many more synonymous (ks) and nonsynonymous (ka) nucleotide substitutions between El Batán 3 and the other two isolates than between the two American isolates (Fig. 3 and Table 1). The ks/ka ratios were 13.1 between El Batán 3 and Sidney 81, 13.0 between El Batán 3 and Type, and 15.3 between Type and Sidney 81 . These values were not significantly different from each other by a Chi square test $(P=0.36)$. In contrast, the ratio of transitions (ts) to transversions (tv) between Type and Sidney $81(\mathrm{ts} / \mathrm{tv}=4.68)$ was significantly different $\left(P<10^{-3}\right)$ from those between El Batán 3 and Sidney 81 or between El Batán 3 and Type (ts/tv = 1.56 for both). The $5^{\prime}$-noncoding region of El Batán 3 was only $69 \%$ identical to the U.S. isolates while the $3^{\prime}$-noncoding region of El Batán 3 shared $89 \%$ (Type) to $91 \%$ (Sidney 81) identity. Both noncoding regions of the American isolates differed from each other by less than $2 \%$.

Pairwise synonymous differences among the three WSMV genomes were examined as a function of nucleotide position by a sliding window procedure (window size 50, step size 10). Thus, potential variation in apparent substitution rates can be viewed along the viral genome. The frequency of silent substitutions was constant over most of the polyprotein open reading frame, with one exception (Fig. 4A). A $400 \mathrm{nt}$ segment of the genome (Sidney $81 \mathrm{nt}$ positions 2745-3150) corresponding to the $3^{\prime}$-end of the $\mathrm{P} 3$ cistron had a striking and statistically significant lower synonymous substitution rate than the rest of the genome. This same region of the WSMV genome also contained few nonsynonymous substitutions (Fig. 3). A similar analysis conducted with five sequences of the potyvirus Potato virus $A$ (PVA) revealed that this distantly related virus also lacked intraspecies variation in the $3^{\prime}$-end of P3 (Fig. 4B), although sequence conservation between 

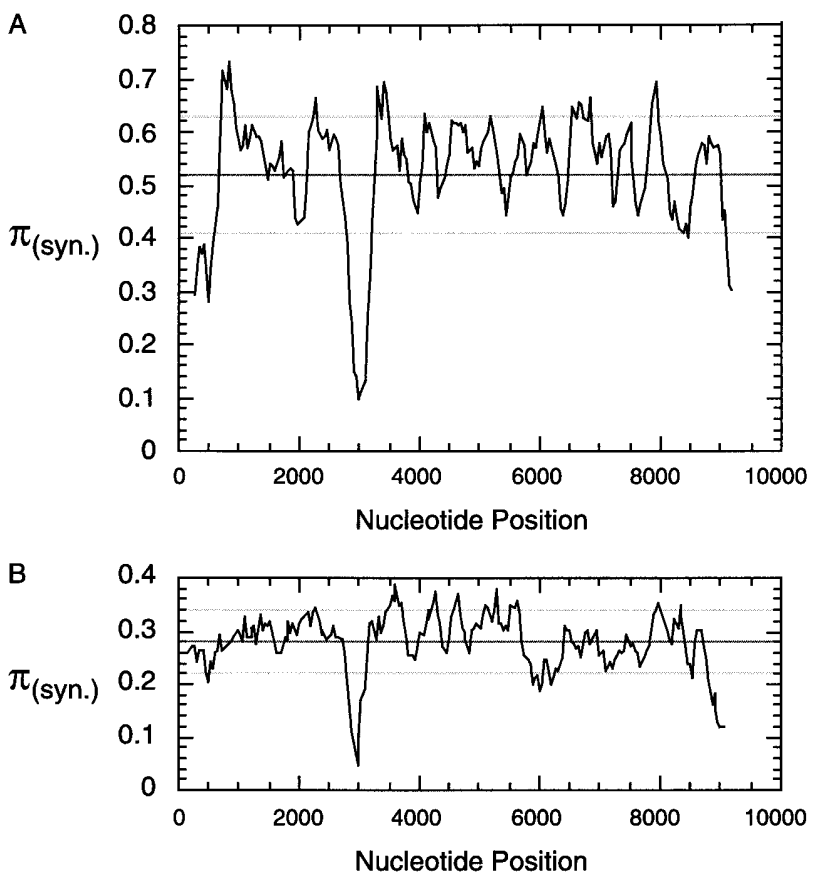

Fig. 4. Distribution of synonymous nucleotide substitutions in the polyprotein open reading frame of A Wheat streak mosaic virus and B Potato virus A. Presented are plots of average pairwise identities $\left[\pi_{(\text {syn. }}\right]$ as a function of nucleotide position calculated using the DnaSP 3.0 program [26]. Black horizontal line indicates the mean; gray horizontal lines denote one standard deviation above and below the mean. Note the lack of intraspecies variation in the region of the genome (nts 2745-3150) corresponding to the $3^{\prime}$-end of the P3 cistron

these two divergent species is low. The same result was obtained when three sequences of Potato virus $Y$ were examined (data not shown). Collectively, this indicates strong selection at the intraspecies level for nucleotide sequence conservation within the $3^{\prime}$-end of the $\mathrm{P} 3$ cistron. Reduced relative substitution rates could be due to several reasons, such as overlapping ORFs or RNA regulatory elements with highly conserved nucleotide sequence requirements. None of the virus sequences examined had ORFs in common in this region other than the polyprotein, and the genome expression strategy of the family Potyviridae make it unlikely that overlapping ORFs are utilized by these viruses. This strongly implies the presence of a species-specific cis-acting RNA regulatory element of as yet unknown function.

American isolates of WSMV show little genetic differentiation, whereas the El Batán 3 strain from Mexico has clearly diverged from the American isolates. The simplest explanation is extended genetic isolation of the two North American populations. It has been suggested that the genomes of some plant RNA viruses might be relatively rigid, where even silent changes are subject to negative selection [11]. The considerable divergence of Type and Sidney 81 from El Batán 3 implies that the WSMV genome is actually quite plastic and able to tolerate many nucleotide substitutions. The predominance of silent substitutions is 
consistent with a period of neutral mutation and genetic drift after the American and Mexican populations separated. Amino acid substitutions have occurred at the same relative rate as synonymous changes (i.e., ks/ka ratios are equivalent for all two-way comparisons), also suggesting a demographic history dominated by stochastic variation rather than positive selection.

Genetic distances among the WSMV strains likely reflect time elapsed since separation from a common ancestor. Tajima [32] proposed that sites in which exactly two nucleotides are present among three gene sequences allow a simple means for testing the molecular evolutionary clock hypothesis. There are 78 such sites for the Sidney 81 sequence and 91 sites for Type, both relative to El Batán 3 and each other. The relevant chi-square test [32] is $\chi^{2}=(91-78)^{2} /(91+78)=1.0$ ( $P=0.317$ for one degree of freedom); thus the molecular evolutionary clock null hypothesis cannot be rejected by our data.

We propose that much of the divergence among strains of WSMV may be explained by drift in which genetic isolation facilitates accumulation and fixation of neutral mutations. Time of isolation then accounts for the amount of divergence between strains. However, selection also is an important factor. Clearly, much of the WSMV genome is constrained by negative selection such that certain regions of the genome retain high sequence identity. The GDD polymerase motif within NIb represents a well known example of sequence conservation likely constrained by negative selection. The P3 mutation cold spot may well represent another example where negative selection acts to conserve a sequence element at the intraspecies level. Drift and selection are not mutually exclusive forces and may occur concurrently. Thus, it is the combined effects of both forces acting simultaneously on different regions of the genome that ultimately determine the extent of divergence within a viral species.

\section{Acknowledgements}

We thank Melissa Morris and Susan Steele for excellent technical assistance and Martin Dickman, John Hammond, Les Lane, and Frank Rabenstein for helpful comments. Mention of proprietary or brand names are necessary to report factually on available data; however, the USDA neither guarantees nor warrants the standard of the product, and the use of the name by USDA implies no approval to the exclusion of others that also may be suitable. This article is in the public domain and not copyrightable. It may be freely reprinted with customary crediting of source.

\section{References}

1. Aleman-Verdaguer M, Goudou-Urbino C, Dubern J, Beachy RN, Fauquet C (1997) Analysis of the sequence diversity of the $\mathrm{P} 1, \mathrm{HC}, \mathrm{P} 3, \mathrm{NIb}$ and $\mathrm{CP}$ genomic regions of several yam mosaic potyvirus isolates: implications for the intraspecies molecular diversity of potyviruses. J Gen Virol 78: 1253-1264

2. Brakke MK (1987) Virus disease in wheat. In: Heyne EG (ed) Wheat and wheat improvement, 2nd ed. ASA-CSSA-SSSA, Madison, pp 585-603

3. Brakke MK, Skopp RN, Lane LC (1990) Degradation of wheat streak mosaic virus capsid during leaf senescence. Phytopathology 80: 1401-1405 
4. Chenault, KD, Hunger RM, Sherwood JL (1996) Comparison of the nucleotide sequence of the coat protein open reading frame of nine isolates of wheat streak mosaic rymovirus. Virus Genes 13: 187-188

5. Choi I-R, French R, Hein GL, Stenger DC (1999) Fully biologically active in vitro transcripts of the eriophyid mite-transmitted wheat streak mosaic tritimovirus. Phytopathology 89: $1182-1185$

6. Choi I-R, Stenger DC, French R (2000) Multiple interactions among proteins encoded by the mite-transmitted wheat streak mosaic tritimovirus. Virology 267: 185-198

7. Djiemboev JT (1956) Disease of hard wheat in North Kazakh, S.S.R. and their control Rev Appl Mycol 37: 344-345

8. Foulad R, Izadpanah K (1986) Identification of wheat streak mosaic virus in Iran. Iran Agr Res 5: 73-84

9. French R, Robertson NL (1994) Simplified sample preparation for detection of wheat streak mosaic virus and barley yellow dwarf virus by PCR. J Virol Methods 49: 93-100

10. Fröhlich K-U (1994) Sequence Similarity Presenter: A tool for the graphic display of similarities of long sequences for use in presentations. Comput Appl Biosci 10: 179-183

11. Gibbs AJ, Keese PL, Gibbs MJ, Garcia-Arenal F (1999) Plant virus evolution; past present and future. In: Domingo E, Webster R, Holland J (eds) Origin and evolution of viruses. Academic Press, New York, pp 263-285

12. Gotz R, Huth W, Maiss E (1995) Molecular analyses of the coat protein region of different viruses on Poaceae belonging to the Potyviridae. Agronomie 15: 491-494

13. Gotz R, Maiss E (1995) The complete nucleotide sequence and genome organization of the mite-transmitted brome streak mosaic rymovirus in comparison to those of potyviruses. J Gen Virol 76: 2035-2042

14. Hall JS, Adams B, Parsons TJ, French R, Lane LC, Jensen SG (1998) Molecular cloning, sequencing, and phylogenetic relationships of a new potyvirus: sugarcane streak mosaic virus, and a reevaluation of the classification of the Potyviridae. Mol Phylogenet Evol 10: $323-332$

15. Hey J, Wakely J (1997) A coalescent estimator of the population recombination rate. Genetics 145: 833-846

16. Janczak C, Lawecki T, Pawlak A (1998) New viruses occurring on wheat plants in Poland. Prog Plant Protect 38: 93-100

17. Kekarainen T, Mertis A, Oruetxebarria I, Rajamaki M-L, Valkonen JPT (1999) Comparison of the complete sequences of five different isolates of Potato virus A (PVA), genus Potyvirus. Arch Virol 144: 2355-2366

18. Lane LC (1986) Propagation and purification of RNA plant viruses. Methods Enzymol 118: 687-696

19. Makkouk KM, Kumari SG (1997) Natural occurrence of wheat streak mosaic virus on wheat in Syria. Rachis 16: 74-76

20. McKinney HH (1937) Mosaic diseases of wheat and related cereals. U.S. Dep. Agric. Circ. 442

21. McNeil JE, French R, Hein GL, Baenziger PS, Eskridge KM (1996) Characterization of genetic variability among natural populations of wheat streak mosaic virus. Phytopathology 86: $1222-1227$

22. Niblett CL, Zagula KR, Calvert LA, Kendall TL, Stark DM, Smith CE, Beachy RN, Lommel SA (1991) cDNA cloning and nucleotide sequence of the wheat streak mosaic virus capsid protein gene. J Gen Virol 72: 499-504

23. Nyitrai A, Gaborjanyi R (1998) Wheat streak mosaic a new virus disease for Hungary. Cereal Res Commun 16: 261-263 
24. Page RDM (1996) TREEVIEW: An application to display phylogenetic trees on personal computers. Comput Appl Biosci 12: 357-358

25. Reshetnik GV, Mishchenko LT, Kolesnik LV, Boiko AL (1996) Detection of wheat streak mosaic virus in some regions of the Ukraine. Mikrobiol Zhurnal 58: 39-45

26. Rozas J, Rozas R (1999) DnaSP version 3: an integrated program for molecular population genetics and molecular evolution analysis. Bioinformatics 15: 174-175

27. Salm SN, Rey MEC, Rybicki EP (1996) Phylogenetic justification for splitting the Rymovirus genus of the taxonomic family Potyviridae. Arch Virol 141: 2237-2242

28. Sanchéz-Sanchéz H, Henry M, Cárdenas-Soriano E, Alvizo-Villasana H (2001) Identification of Wheat streak mosaic virus and its vector Aceria tosichella Keifer in Mexico. Plant Dis 85: 13-17

29. Slykhuis JT, Bell W (1963) New evidence on the distribution of wheat streak mosaic virus and relation of isolates from Rumania, Jordan, and Canada. Phytopathology 53: 236-237

30. Stenger DC, Hall JS, Choi I-R, French R (1998) Phylogenetic relationships within the family Potyviridae: wheat streak mosaic virus and brome streak mosaic virus are not members of the genus Rymovirus. Phytopathology 88: 782-787

31. Strimmer K, von Haeseler A (1996) Quartet puzzling: a quartet maximum likelihood method for reconstructing tree topologies. Mol Biol Evol 13: 964-969

32. Tajima F (1993) Simple methods for testing the molecular evolutionary clock hypothesis. Genetics 135: 599-607

33. Thompson JD, Gibson TJ, Plewniak F, Jeanmougin F, Higgins DG (1997) The ClustalX windows interface: flexible strategies for multiple sequence alignment aided by quality analysis tools. Nucleic Acids Res 24: 4876-4882

34. Xie H, Wang Z, Li W, Ni S (1982) Studies of wheat streak mosaic virus in Xinjiang. Acta Phytopathol Sin 12: 7-12

Authors' address: Dr. D. C. Stenger, USDA-ARS, 344 Keim Hall, University of Nebraska, Lincoln, NE 68583, U.S.A.

Received April 10, 2000 